\title{
THE ON- AND OFF-STATE GENERATIONS IN THE CASE OF THE THICK ACCRETION DISK AND UNDEFINED PRECESSION PERIOD. 3-D NUMERICAL HYDRODYNAMICAL SIMULATIONS IN ACCRETION DISK IN MICROQUASAR CYG X-1
}

\author{
V.V. Nazarenko \\ Astronomical Observatory, Odessa National University, \\ Shevchenko Park, Odessa, 65014, Ukraine, nazaret@te.net.ua
}

\begin{abstract}
In the present work we have computed the thick accretion disk (the accretion disk with radiation pressure) for the case of undefined precession over very long time (more than 14 precession periods). The calculations show that in this case the origin of the ON-states and OFF-states are strong irregular in time. The calculations also show that the jet's launches are taking place very suddenly over $7 \div 20$ minutes of the orbital time. The jet's launches over OFF-states occurs discrete as it is observed in real microquasars.
\end{abstract}

АНОТАЦЯ. В представленій роботі ми виконали обчислення товстого акреційного диску (акреційного диску з радіативним тиском) для випадку невизначеної прецесії протягом довгого часу (більше чотирнадцяти прецесійних періодів). Обчислення показали, що в цьому випадку генерація виключених та включених станів є дуже іррегулярними з часом. Результати розрахунків також показали, що запуск джет виникає раптово протягом семи - двадцяти хвилин орбітального часу. Запуск джет протягом виключених станів в наших обчисленнях виникає дискретно, що відповідає спостереженням в реальних мікроквазарах.

Keywords: Stars: binaries - stars: jets - methods: numerical - hydrodynamics.

\section{Introduction}

In the present research we have continued to simulate the ON- and OFF-states generations on the base of microquasar Cyg X-1 by the methods of 3-D numerical hydrodynamics (Nazarenko \& Nazarenko, 2014, 2015, 2016, 2017; Nazarenko V.V., 2018). The present work is devoted to the thick accretion rates simulations on the base of classical microquasar Cyg $\mathrm{X}-1$. The goal of the present research is to compute the donor's wind, one-point-stream formation, its motion in Roche lobe of accretor, the thick accretion disk formation and it's slaved precession for a irregular precession period. To calculate the thick accretion disk we will use in the calculations the computations of the $\mathrm{z}$-direction radiation pressure in the disk volume.

\section{The numerical algorithm}

The description of the numerical algorithm in use in details is given in our previous works (Nazarenko \& Nazarenko, 2014, 2015, 2016, 2017; Nazarenko V.V., 2018). Shortly, this algorithm is as follows: to resolve the non-stationary Euler's hydrodynamical equations we have used the astrophysical variant of "largeparticles" code by Belotserkovsky and Davydov (Belotserkovskii O.M., Davydov Yu.M., 1982); to simulate one-point-stream we use the donor's atmosphere model that in turn is constructed on the base Kurucz's grid (Kurucz R.L., 1979) with the donor's parameters; we use the free-flow boundary conditions allowing to a gas to flow freely via the calculation area boundaries; to calculate mass flow real temperature we use the radiation cooling explicitly (Cox D. P., Daltabuit E., 1971). In the present calculation we use the rectangular coordinate system centred on the donor's centre. We have adopted the donor's mass to be equal to 40 solar mass and the accretor's mass to be equal to 10 solar mass. The precession period in the present simulations is about of 8 orbital periods. Hereafter all the distances will be given in units of the orbital separations; the average volume disk specific viscosity and the average volume disk specific angular momentum will be given in units of $V_{0} A$, where $V_{0}$ is the orbital speed and $\mathrm{A}$ the orbital separation; the time are given in the unit 
such that $2 \pi$ is equal to orbital period.

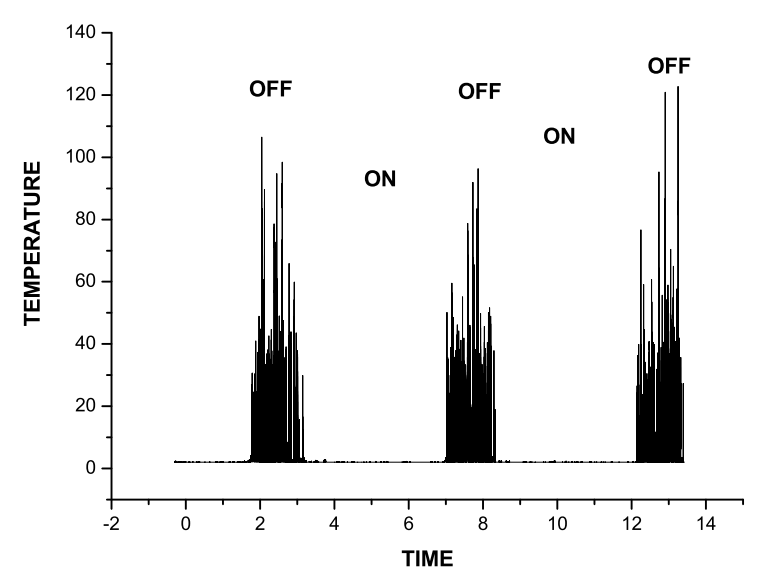

Figure 1: The central disk's temperature over the orbital plane versus time.

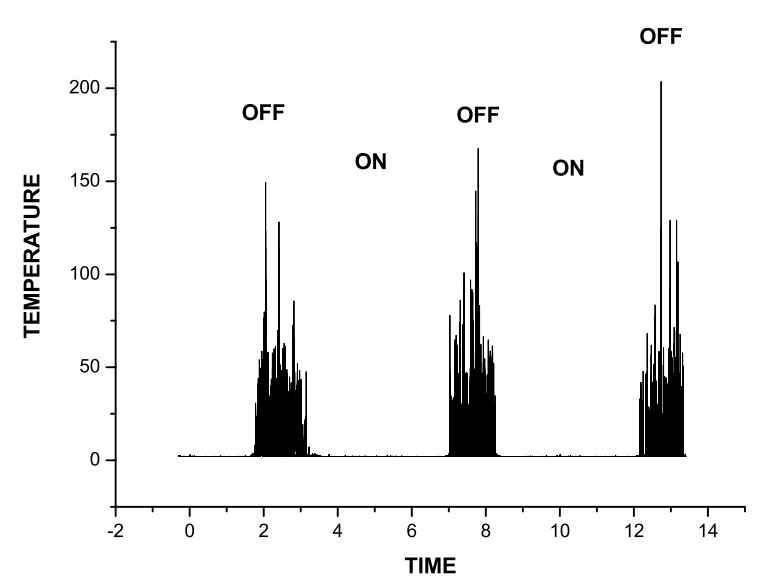

Figure 2: The central disk's temperature below the orbital plane versus time.

To calculate the radiation pressure in disk we will use the radiation model in the approach of the optically thin layers and we will use the following formula of radiation pressure: $F_{R A D}=\alpha \mathrm{H} / \mathrm{C}$, where $\alpha$ is coefficient of Thomson scattering, $\mathrm{H}$ is the radiation flux which is calculated in terms of thermodynamical equilibrium, $\mathrm{C}$ is the light speed.

We denote the $\mathrm{X}, \mathrm{Y}$ and Z-coordinate of the accretor1 as $X_{A C C 1}, Y_{A C C 1}$ and $Z_{A C C 1}$. The time evolution of these values are defined by the relations:

$Z A 19=0.15 Z B 19=0.15 Z C 19=0.15$

TET222 $=\mathrm{TET} 222+\mathrm{DT} / 8.0$ where DT is time step, given in units described above; the number 8.0 is the quantity of the orbital periods in the one precession period. TET $222_{I N I T}=-14.846$, since we start the precession motion on time 0.00 and before the precession starting we must form accretion disk and to make it in stationary state.

$$
\begin{aligned}
& Z A 199=Z A 19 *(\cos (T E T 222 / Z A 221))^{Z A A 2221}, \\
& Y_{a c c 1}=Z A 199 *(\sin (T E T 222 / Z A 21))^{Z A A 19}, \\
& Z B 199=Z B 19 *(\cos (T E T 222 / Z B 221))^{Z B B 2221}, \\
& Z_{a c c 1}=Z B 199 *(\cos (T E T 222 / Z B 21))^{Z B B 19}, \\
& Z C 199=Z C 19 *(\cos (T E T 222 / Z C 221))^{Z C C 2221}, \\
& X_{a c c 1}=1.0-Z C 199 *(\cos (T E T 222 / Z C 21))^{Z C C 19},
\end{aligned}
$$

In the formulas written above the values ZA19, ZB19, ZC19, ZA21, ZB21, ZC21, ZAA19, ZBB19, ZCC19, ZA221, ZB221, ZC221, ZAA2221, ZBB2221, ZCC2221 are the parameters. These parameters are in the present calculations equal correspondingly to the following magnitudes: $0.15,0.15,0.15,4.50,2.89$, 5.19, 1.0, 1.0, 1.0, 99999999.0, 99999999.0, 99999999.0, $0.0,0.0,0.0$.

\section{The results}

We have begin our simulations with the start of the precession motion and have continued it over 14 precession periods to show our on-off state model on relatively long time scale. The key parameters in the present calculations are the central disk's temperature over and below orbital plane. We show the time dependencies of these parameters in Fig.1 and Fig.2. As it is led from these figures the off-states are arising every 5 precession periods and the beginning and end of these states occurs very rapidly over $7 \div 20$ minutes of orbital plane. The time intervals of off-states are about of one precession period. Thus, it means, that the jet's launches take place practically very suddenly in respect to the off-state durations. The comparison both Fig.1 and Fig.2 pictures shows that they are practically identical to a each other. It means that in the present simulations two armed jets are simulated. To show the OFF-state structure in more details we show time dependence of central disk's temperature on the vicinity of $7 \div 8$ precession period (see Fig. 3 ). As it is led from this figure the OFF-state structure is consisting of the partial pics and this result is in good accordance with observation (Fender et al. 2003, 2004) The time dependencies of jet's velocities in the disk's centrE over and below orbital plane are showing in Fig. 4 and Fig.5. As it is led from these figures the jet's launches are beginning suddenly and velocities in off-states are reaches $2000 \div 2500 \mathrm{~km}$ per second. Such the relatively small jet's velocity is explained by the circumstance that jet's launches in our calculations occurs on the highness of 5000 shvarchild radius instead of real 50 ones. The details of the jet's launches on the $7 \div 8$ precession period is seen in Fig.6. From 


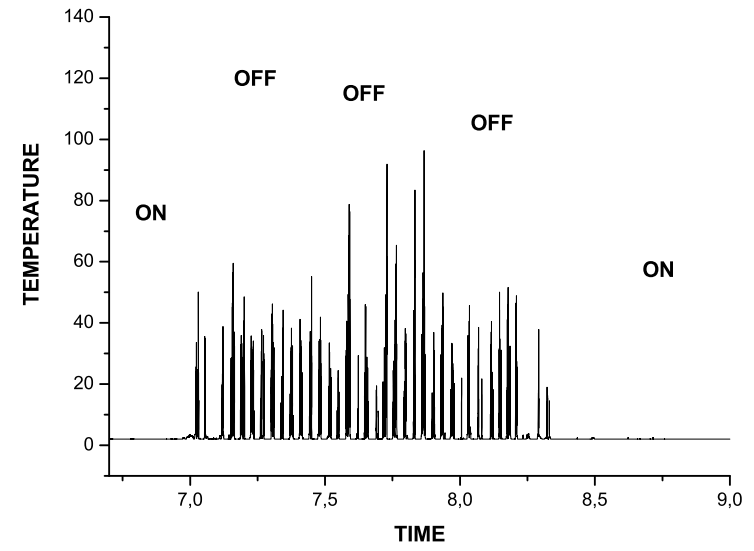

Figure 3: The central disk's temperature over the orbital plane versus time in details for the OFF-state on $7 \div 8$ precession periods.

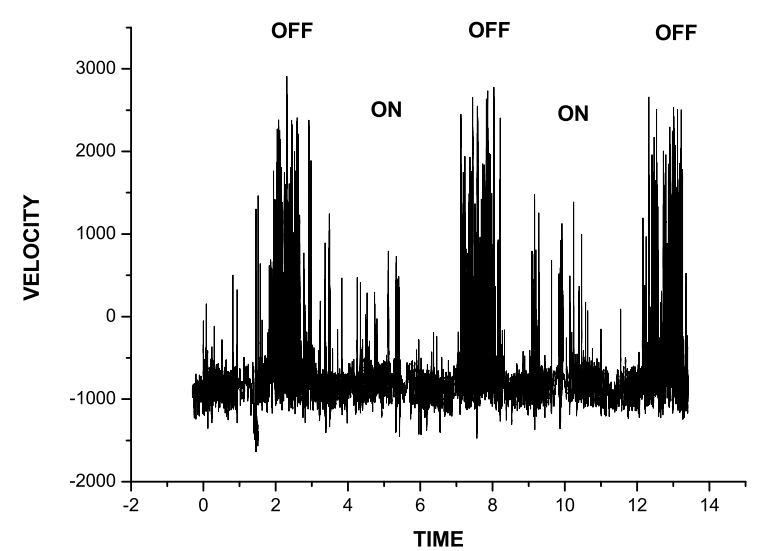

Figure 4: The central disk's temperature over the orbital plane versus time.

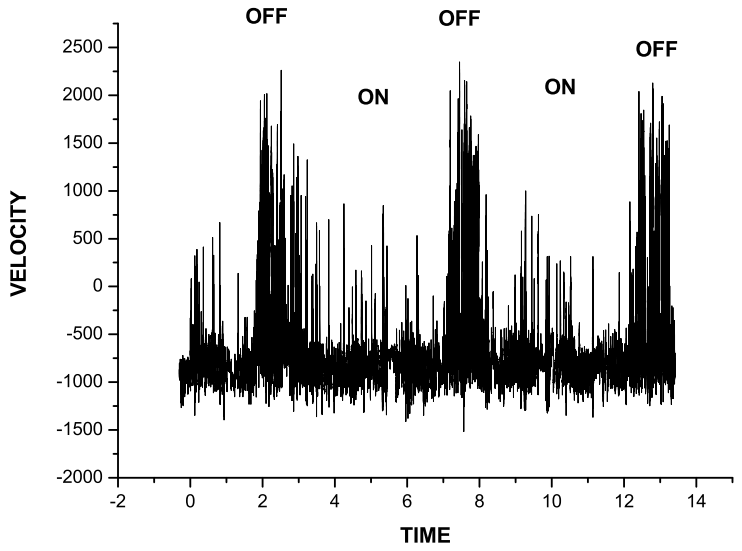

Figure 5: The central disk's velocity below the orbital plane versus time.

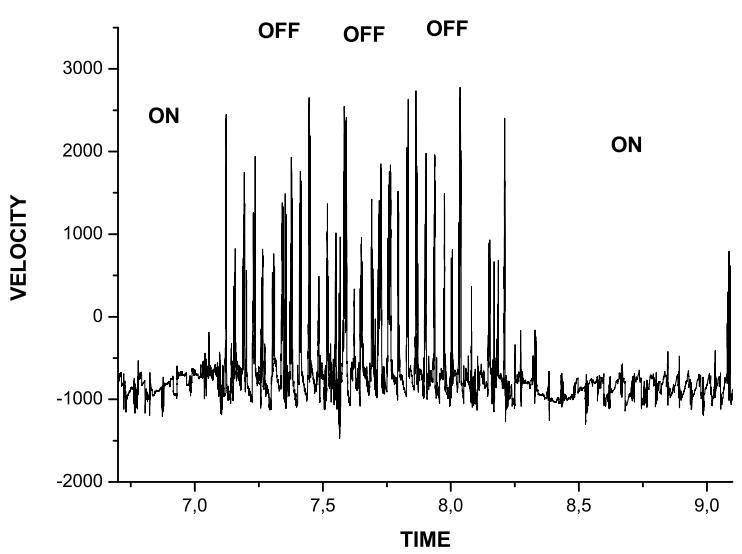

Figure 6: The central disk's velocity over the orbital plane versus time in details for the OFF-state on $7 \div$ 8 precession periods. 

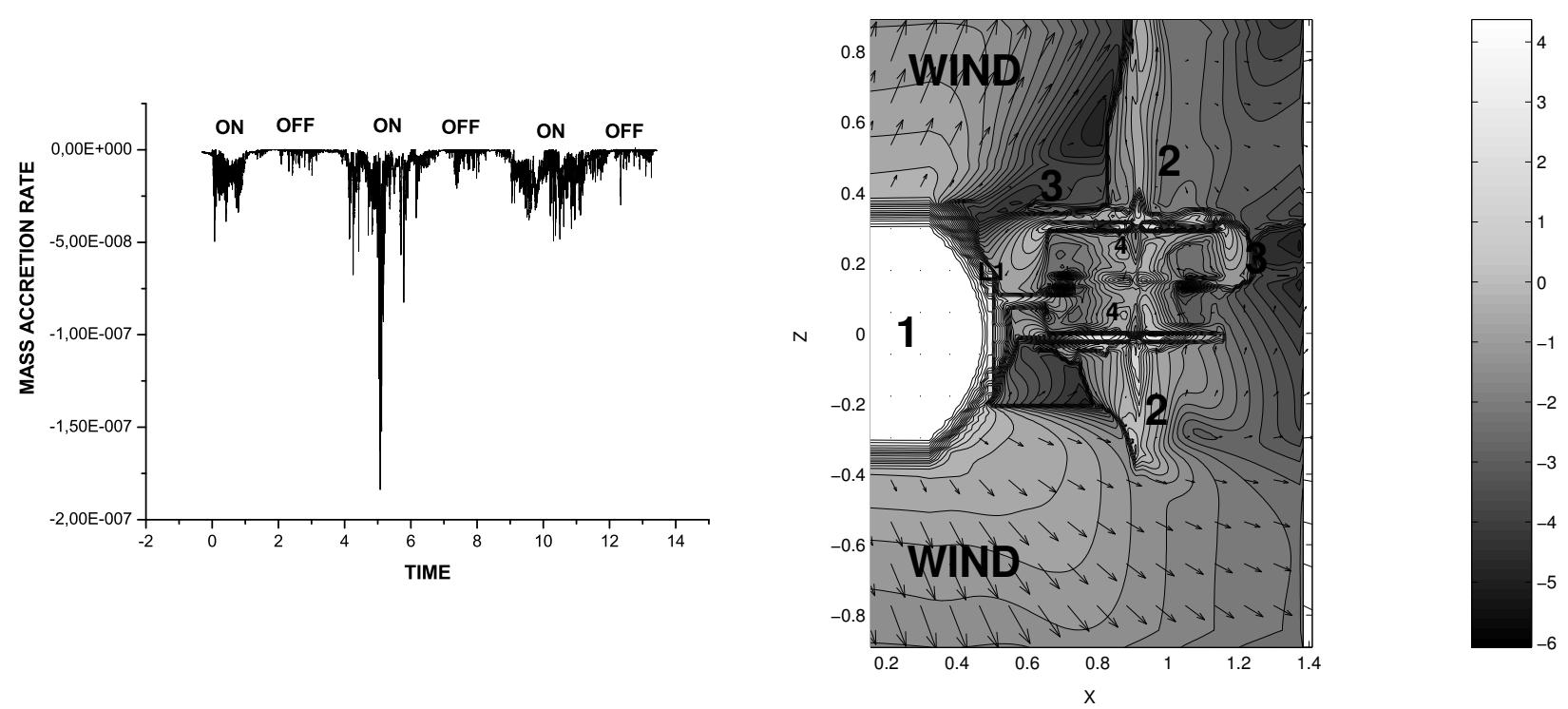

Figure 7: The mass accretion rate versus time.

Figure 9: The cross-section of the calculation area by the $\mathrm{Z}-\mathrm{X}$ plane.
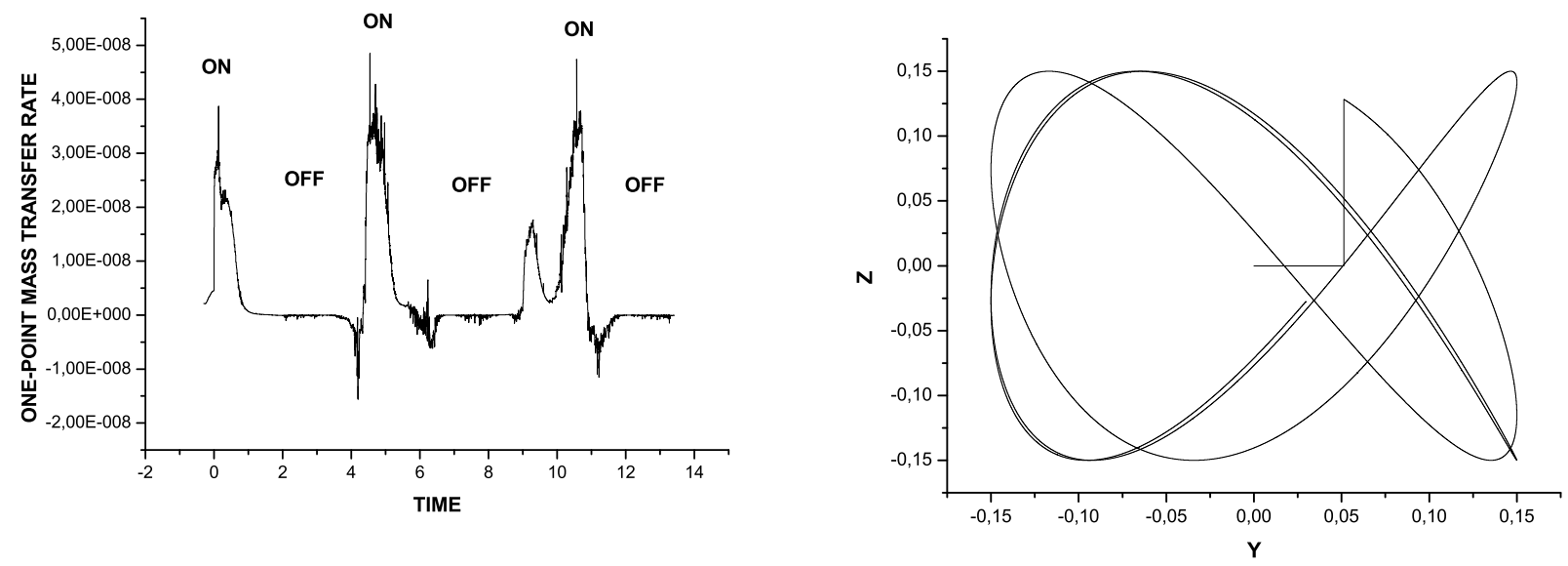

Figure 8: The mass transfer rate versus time.

Figure 10: The accretor-1 motion in the Z-Y plane. 
these figure it is good seen that the jet launches over off-state is in the view of the partial picks also as the central disk's part temperature structure. The mass accretion rate near the disk centre time dependence is shown in Fig 7. As it is led from these figure this value is anticorellated with temperature in the disk's centre. The time dependence of mass transfer rate via one-point is shown in Fig.8. These figure shows the strong variations of this value with time (here we must point out that the position of the point via which we have calculated the mass transfer rate in the present research was fixed in space and time and was corresponding to $X_{a c c 1}=1.0$ ). Namely these variations explains the off- and on-states generation in the present research. Indeed, over high mass transfer rate value the density in the disk centre is high and the radiation cooling results in the low temperature in the disk's centre. On contrary, over low mass transfer rate the density in the disk's centre is strong decreased and radiation cooling is not affective in this case. It results in instantaneously heating the space near the disk centre and jets are launching in this moment. The one-point mass transfer rate varieties are in turn resulting from a precession motion. Such the mechanism of off- and on-states generation is working in the present research. To illustrate the vertical structure of the thick accretion disk we show this structure in Fig.9 in which the $\mathrm{z}-\mathrm{x}$ plane cross-section of the calculation area is plotted. In this figure we may see the donor (the number 1 ), one-point stream $\left(L_{1}\right)$, the jets (the number 2), the donor's wind (wind), the thick accretion disk (the number 3) and the funnel along the disk rotation axis (the number 4 ). To illustrate the accretor- 1 motion in the present precession model we show this motion in Fig. 10.

\section{Summary and conclusions}

The present calculations show that in the case of undefined precession period jointly with the variable binary separation (on time scale of precession periods) the generation of off- and on-states begins to be not every precession period and off-states are arising approximately every 5 precession periods. The jet launches occur very rapidly over $7 \div 20$ minutes of orbital plane. This is very short time scale since the off-state time interval is about of 1 precession period. As it is led from the present research the off-on-states generations are explained by the precession motion of the disk. The explanation of the jet origination that is working in the present research is very convenient for the radiation-driven jets and it is in bade agreement with the magnetically-driven jets.
Finally we may conclude that our jet launch model are in very good accordance with the observations (Mirabel \& Rodriquez, 1999; Stirling et al., 2001; Fender et al., 2003, 2004; Migliari \& Fender, 2006) through the following points:

1. The first is that jet launch in our model is occur practically instantaneously about of $7 \div 20$ minutes of orbital plane.

2. The second is the anticorrelation between mass accretion rate and the central disk's temperature.

3. The third is the jet time structure that is in the view of the partial pics not connected with a each other.

\section{References}

Belotserkovskii O.M., Davydov Yu.M.: 1982, "The large particles code in gas dynamics", Moscow: Nauka, 391.

Cox D.P., Daltabuit E.: 1971, ApJ, 167, 113.

Fender R.P., Gallo E., Jonker P.: 2003, MNRAS, 343, L99.

Fender R.P., Belloni T., Gallo E.: 2004, MNRAS, 355, 1105.

Kurucz, R.L.: 1979, ApJ.Suppl.Ser., 40, 1.

Mirabel L.F. \& Rodriquez L.F.: 1999, Ann. Rev. Astron. Astrophys., 37, 409.

Migliari S. \& Fender P.: 2006, MNRAS, 366, 79.

Nazarenko V.V., Nazarenko S.V.: 2014, Odessa Astron. Publ., 27, 137.

Nazarenko V.V., Nazarenko S.V.: 2015, Odessa Astron. Publ., 28, 171.

Nazarenko V.V., Nazarenko S.V.: 2016, Odessa Astron. Publ., 29, 82 .

Nazarenko V.V., Nazarenko S.V.: 2017, Odessa Astron. Publ., 30, 113.

Nazarenko V.V.: 2018, Odessa Astron. Publ., 31, 90. Stirling A.M. et al.: 2001, MNRAS, 327, 1273. 